\title{
Expulsion : genèse et pratique d'un contrôle en Allemagne (partie 2)
}

\section{Frank Paul Weber}

\section{OpenEdition \\ Journals}

\section{Édition électronique}

URL : http://journals.openedition.org/conflits/625

DOI : $10.4000 /$ conflits. 625

ISSN : $1777-5345$

Éditeur :

CCLS - Centre d'études sur les conflits lilberté et sécurité, L'Harmattan

Édition imprimée

Date de publication : 15 octobre 1996

ISSN : 1157-996X

\section{Référence électronique}

Frank Paul Weber, «Expulsion : genèse et pratique d'un contrôle en Allemagne (partie 2) », Cultures \& Conflits [En ligne], 23 I automne 1996, mis en ligne le 15 mars 2006, consulté le 30 mars 2021. URL : http://journals.openedition.org/conflits/625 ; DOI : https://doi.org/10.4000/conflits.625

Ce document a été généré automatiquement le 30 mars 2021

Creative Commons License 


\title{
Expulsion : genèse et pratique d'un contrôle en Allemagne (partie 2)
}

\author{
Frank Paul Weber
}

La rétention en République Fédérale d'Allemagne

Le décret sur la police des étrangers de 1938 demeura le principal texte législatif régulant le séjour et l'entrée d'étrangers en République Fédérale d'Allemagne jusqu'à la première loi, votée en 1965, sur les étrangers [Ausländergesetz] de la R.F.A.

Le règlement portant sur le placement en détention d'étrangers préalablement à leur expulsion était maintenu dans la loi sur les étrangers [Ausländergesetz] du 28 avril 1965. La durée maximale de cette détention pour "garantie de l'expulsion » [zur Sicherung der Abschiebung] fut fixée. L'article 16 de la loi énonçait qu' « (2) un étranger est à placer en centre de rétention [Abschiebungshaft], lorsque la détention est nécessaire à la garantie [Sicherung] de l'expulsion. La détention en vue de l'expulsion peut être ordonnée pour une durée de six mois et prolongée pour une durée totale d'un an $»$.

Les législateurs indiquèrent deux motifs principaux, qui, à leurs yeux, justifiaient le recours aux centres de rétention. D'une part, la détention en vue de l'expulsion était nécessaire afin de prévenir tout passage de l'étranger dans la clandestinité : «les autorités des étrangers [Ausländerbehörde] doivent, le cas échéant, disposer de cette restriction à la liberté [i.e. le placement en détention], pour pouvoir exclure un étranger du territoire fédéral. Une grande partie des étrangers n'a pas d'attaches fixes à un lieu de résidence précis sur le territoire fédéral, ils peuvent donc facilement se soustraire à l'arrestation des autorités " ${ }^{1}$. D'autre part le placement en centre de rétention était nécessaire car l'admission [Übernahme] de la personne concernée par un autre Etat exigeait une certaine durée: «une expulsion dépendant toujours du consentement d'un autre Etat à admettre l'expulsé et étant souvent encadrée par des délais fixes, il importe aussi dans le cadre de l'expulsion, que l'étranger soit durant ces délais à la disposition des autorités des étrangers ${ }^{2}$.

Les clauses de la loi sur les étrangers de 1965 témoignaient déjà du paradoxe constant $\mathrm{du}$ statut juridique des étrangers. Une suspension provisoire de l'expulsion 
("Duldung», art.17) ainsi que le dépôt d'un recours contre l'ordre de placement en centre de rétention étaient certes rendus possibles, alors que le décret sur la police des étrangers de 1938 excluait explicitement tout recours juridique contre le renvoi et le placement en centre de rétention ${ }^{3}$.

Mais, dans le même temps, le placement en centre de rétention devenait une obligation, là où le décret adopté sous le Troisième Reich n'en faisait qu'une possibilité. En comparant les textes de 1938 et de 1965, on peut en effet noter ce changement avec la substitution du verbe " pouvoir » par le verbe « devoir » [en fait « être »].

Depuis le placement en centre de rétention est réglementé par l'article 57 de les lois sur les étrangers du 9 juillet 1990 et du 30 juin 1993.

Il existe deux formes de placement en centre de rétention: "la détention de préparation " [Vorbereitungshaft] et «la détention de sûreté » [Sicherungshaft]. La Vorbereitungshaft est ordonnée afin de préparer un arrêté d'interdiction du territoire [Ausweisung], dans le cas où la personne n'a pas encore été enjointe de quitter le territoire [Ausreisepflicht] et où il ne peut être statué immédiatement à propos de l'arrêté d'interdiction du territoire. Cette "détention de préparation » peut durer jusqu'à six semaines. A l'encontre d'étrangers en situation irrégulière est prononcée beaucoup plus souvent la Sicherungshaft] et ce par un juge de détention [Haftrichter] sur demande des autorités des étrangers auprès du tribunal de première instance [Amtsgericht]. La détention qui en découle peut aller jusqu'à dix-huit mois ${ }^{4}$. Dans la loi de 1965, la « détention de sûreté » ne pouvait excéder un an.

La rétention comme peine de facto

Le fossé séparant les pratiques de placement en centre de rétention de ses fondements juridiques abstraits n'est peut-être jamais aussi manifeste que dans la comparaison avec le droit pénal et le régime pénitentiaire.

Si la loi sur les étrangers énumère en effet les conditions nécessaires au placement en centre de rétention, elle ne contient toutefois aucune disposition règlementant le régime pénitentiaire propre au centre de rétention [Abschiebungshaftvollzug] ${ }^{5}$.

A l'automne 1995, le Land de Berlin fut contraint, d'adopter une «loi portant sur la détention dans le but de l'expulsion » [Gesetz über den Abschiebungsgewahrsam] à la suite d'un jugement du tribunal administratif de Greifswald ayant constaté qu'« aucun fondement juridique suffisant - au titre des exigences constitutionnelles en matière de loi - n'existe pour le fonctionnement du centre de rétention de la police berlinoise ${ }^{6}$.

Par ailleurs, la détention en centre de rétention, bien que n'étant pas en fait une sanction pénale, correspond à une mesure punitive.

Lorsqu'un étranger placé en centre de rétention refuse d'apporter son concours aux «mesures d'établissement du passeport » [Paßbeschaffungsmaßnahmen] - c'est-à-dire lorsqu'il n'a pas rempli, ou de façon incomplète, un formulaire de demande d'établissement de documents remplaçant le passeport - alors les autorités des étrangers l'informent qu'il risque, en conséquence, la durée maximale de détention (18 mois). Le séjour en centre de rétention devient alors en définitive indépendant de la possibilité exécuter l'expulsion, bien que, d'après la loi, « la détention de sûreté n'est pas autorisée, lorsqu'il est attesté, pour des raisons que l'étranger ne peut plaider, que l'expulsion ne peut être exécutée dans les trois prochains mois» ( $\$ 57$. Abschiebungshaft, Abs. 2, S.5). 
Le Ministère de l'Intérieur du Land de Berlin rapportait ainsi ouvertement fin 1994 : « si la non-expulsion est jusque-là due à l'absence de passeport, lorsque l'étranger se refuse à participer à l'établissement de son passeport on considère qu'il a empêché l'expulsion. Le fait que, sur la base de l'expérience antérieure, jusqu'à l'établissement d'un passeport valide la [détention] dure encore longtemps ne joue ici aucun rôle. La loi prévoit que des difficultés de cet ordre peuvent retarder l'expulsion et que, dans de tels cas, la durée maximale de dix-huit mois s'applique » ${ }^{7}$.

Le placement en centre de rétention correspond indirectement à une peine, puisque la détention a généralement lieu dans les conditions du régime pénal. Dans douze des seize Bundesländer allemands, le placement en centre de rétention s'effectue dans des maisons d'arrêts [Justizvollzugsanstalt]. Ce sont les instructions contenues dans la loi d'exécution des peines qui sont appliquées au séjour en centre de rétention. Même dans les Länder où le placement en centre de rétention est resté du ressort du Ministère de l'Intérieur (du Land), comme par exemple à Berlin, les règlements se conforment à ce qui est prescrit pour les prisons «classiques»: «Pour le placement en centre de rétention s'appliquent les articles 3 à 108 et 173 à 175 de la loi d'exécution des peines du 16 mars 1976 (...) (2). Le centre de rétention doit, autant que possible, permettre aux personnes détenues [Abschiebehäftlingen] de travailler. Les détenus, qui feront usage de cette possibilité, seront rémunérés au terme de l'art. 43 de la loi d'exécution des peines et de ses règlements annexes ${ }^{8}$. Que les étrangers placés en centre de rétention et les criminels/délinquants soient ainsi mis au même rang est particulièrement préoccupant, sachant que la peine dans sa conception moderne présuppose que quelque chose doit être corrigé ${ }^{9}$, ce qui, chez les étrangers ne pourrait être que leur irrévocable " altérité d'étrangers ».

Le temps de la rétention

Le temps a deux fonctions en centre de rétention. D'une part il comprend la durée qui est nécessaire pour établir l'identité de l'étranger détenu et lui délivrer les documents correspondants (passeport, laisser-passer...). D'autre part le temps sert à essayer de modifier le comportement de la personne détenue vis-à-vis de son expulsion, afin que, par exemple, il aide à l'organisation de sa propre expulsion, en donnant, par exemple, des indications sur sa personne (état-civil, pays de transit...). Les autorités des étrangers espèrent éventuellement ainsi un découragement de l'étranger.

D'une certaine façon, il y a aussi, dans le centre de rétention, cette « économie interne d'une peine qui, si elle sanctionne le crime, peut se modifier (s'abrégeant ou, le cas échéant, se prolongeant) selon que se transforme le comportement du condamné ${ }^{10}$. La durée du placement en centre de rétention d'un étranger est en effet souvent fonction de sa participation ou non à l'établissement de son passeport [Paßbeschaffungsmaßnahmen], permettant ensuite de l'expulser.

Lorsque cette stratégie ne fonctionne pas, cela peut déboucher sur un blocage : la durée maximale de détention autorisée s'approchant sans toutefois, pour les autorités des étrangers, la moindre perspective d'exécuter l'arrêté d'expulsion. Souvent la seule issue est celle de l'« illégalité décrétée ». Les étrangers en centre de rétention, qui ont fait une demande de passeport complète mais toujours sans succès après six mois, doivent en effet, d'après la loi, être relâchés du centre de rétention. Ne disposant d'aucun papier d'identité, les autorités des étrangers leur délivrent un étrange document qui indique le caractère irrégulier de leur séjour en Allemagne mais ne leur permet évidemment pas d'entrer dans un autre pays. De plus ce type de document ne 
semble pas les protéger contre une nouvelle arrestation pour séjour irrégulier (lors d'un simple contrôle de police) et donc, à terme, contre un retour au même centre de rétention pour une toujours aussi hypothétique expulsion. Dans ces cas, encore en petits nombres mais croissants, il semble que l'administration compétente décide et entérine elle-même la clandestinité de migrants, en refusant parallèlement de leur offrir le moindre statut juridique en Allemagne (sursitaire,...etc.).

"Lorsque des réfugiés interdits de séjour sur le territoire, détenus en centre de rétention pour cause d'absence de papiers d'identité, sont relâchés, il existe alors pour l'office des habitants -Section Etrangers LandeseinwohneramtAusländerangelegenheiten (Ausländerpolizei) deux catégories d'êtres humains : ceux que les autorités considèrent comme s'occupant de leurs papiers et qui donc régulièrement obtiennent des convocations. Ceux qui, d'après cette administration, ne font pas suffisamment d'efforts pour obtenir des papiers d'identité, reçoivent à leur libération une "attestation de passage de la frontière " ["Grenzübertrittsbescheinigung»]. Cette attestation indique que la personne mentionnée séjourne illégalement sur le territoire. Aucune de ces personnes libérées du centre de rétention [dans ces conditions] ne peut, en l'absence de papiers, quitter l'Allemagne. Aucune n'obtient le sursis [Duldung] correspondant à cette situation ${ }^{11}$.

Lieux de rétention

De même que le temps joue un rôle central dans le placement en centre de rétention, l'espace est, dans certains cas, une variable largement utilisée par les administrations responsables de la rétention.

Des centres de rétention comme Büren (Rhénanie du Nord-Westphalie), Prötzel (Brandebourg) ou même le nouveau bâtiment utilisé à Berlin (Grünau) sont, par exemple, situés à la périphérie des zones urbaines (dans les deux premiers cas en pleine forêt), dans des lieux difficilement ou non accessibles par des transports publics. Les visites de parents ou amis en sont d'autant plus compliquées, voire coûteuses. Mais cet isolement signifie aussi qu'il est encore plus improbable que des avocats viennent «se perdre » dans ces lieux éloignés ${ }^{12}$.

Rationaliser la rétention et l'expulsion

Les pratiques du placement en centre de rétention suscitent, comme toutes les pratiques sociales, une certaine rationalisation.

A Berlin, le Sénat justifia ainsi la construction d'un nouveau centre de rétention à Köpenick (Grünau) devant permettre l'augmentation des capacités de détention de 176 à environ 400 "places»: «cela permet un emploi du personnel [de surveillance] fondamentalement plus rationnel, l'entreprise [Betrieb] est ainsi aussi considérablement moins coûteuse ${ }^{13}$. Des réflexions de cette sorte n'étonnent guère dans le contexte d'ensemble du dispositif d'expulsion. L'idée que l'expulsé supporte luimême les frais de sa propre expulsion est, par exemple, contenue dans les articles 82 (débiteur; dépôt de garantie) et 83 (Etendue de la responsabilité financière, prescription) de la loi sur les étrangers. Le gouvernement du district de Hanovre avait même, un temps, décidé, que les étrangers placés en centre de rétention devaient euxmêmes payer pour leur séjour dans la maison d'arrêt ${ }^{14}$.

Cette exigence de rationalisation intervient dans différents ensembles du dispositif d'expulsion: le gouvernement allemand a ainsi justifié un récent voyage coûteux du Ministre allemand des Affaires étrangères à Addis-Abeba par « les progrès enregistrés 
dans les discussions sur la réadmission de plus d'un millier de demandeurs d'asile déboutés ». Il était suggéré, de façon implicite, que les frais de ce voyage de 48 heures avaient été compensés par l'argent économisé sur les dépenses du séjour en Allemagne de ces demandeurs d'asile déboutés ${ }^{15}$.

\section{LES DROITS DE RECOURS}

L'expulsion des étrangers comme pratique apparaît en définitive comme le produit d'une juridicisation croissante du rapport aux étrangers, qui, à l'aide de catégories juridiques (droit de la nationalité...), a achevé la différenciation, l'isolement des étrangers vis-à-vis des "nationaux » et donc défini, circonscrit un groupe hétérogène de personnes susceptibles de ne pas bénéficier du principe de non-expulsion de ses propres ressortissants.

Après 1945, cette "insertion " des étrangers dans la sphère juridique s'effectua parallèlement au développement de droits octroyés aux individus, tout comme sous Weimar la régulation juridique du séjour des étrangers évolua conjointement à la juridicisation de la sphère du travail.

En Allemagne de l'Ouest, tout comme ailleurs en Europe et en Amérique du Nord, la période d'après-guerre est, dans ce contexte, caractérisée par une apparition et une solidification des droits de recours juridiques, dont bénéficient également les étrangers.

La question de l'expulsion apparaît comme un des domaines privilégiés de l'immigration où la tension entre les règles du marché et les droits individuels, telle que James Hollifield l'a exposé avec l'idée d'un " paradoxe du libéralisme ", émerge au grand jour. Dans ce contexte du "paradoxe du libéralisme», avec d'une part «la volonté des gouvernements (et des entreprises) de définir le travailleur étranger comme un bien, qui peut être contrôlé, réparti et géré selon les règles du marché dans le cadre d'un système international de libre échange " ${ }^{16}$ et, d'autre part, les droits de recours juridiques dont disposent les étrangers, l'expulsion de travailleurs étrangers n'est plus aussi aisément justifiable à partir des seules considérations de marché du travail (absence de travail, notamment pendant l'hiver ou la protection du marché du travail national), comme cela était le cas sous l'Empire wilhelmien et la République de Weimar.

Durant ces deux périodes (et partiellement lors du recrutement de travailleurs immigrés dans les années 1950 et 1960) cette définition du travailleur étranger comme un bien semble avoir prévalu, les étrangers ne disposant pas encore de " procédures qui limitent et circonscrivent la capacité du pouvoir administratif de l'Etat ${ }^{17}$.

Ces droits de recours, constituant «la première ligne de défense entre les individus (surtout les étrangers qui sont souvent parmi les plus marginalisés) et l'Etat " ${ }^{18}$, sont le principal trait saillant de l'évolution du dispositif d'expulsion depuis 1945 et ce, par opposition à l'agencement antérieur (Empire wilhelmien, Weimar et dictature nazie). Là où la législation sur les étrangers de la République de Weimar ${ }^{19}$ et du Troisième Reich ${ }^{20}$ excluait de pouvoir faire appel d'une décision d'expulsion devant un tribunal administratif indépendant, la loi sur les étrangers de 1965 et ses versions modifiées ultérieures prévoyait un semblable recours.

Ainsi fut écarté «l'îlot de Polizeistaat» (la formule est du juriste Ernst Isay) que constituait la législation sur les étrangers depuis l'Empire, pour l'inscrire dans le cadre de l'Etat de droit [Rechtsstaat]. 
Plus particulièrement en ce qui concerne l'expulsion, la législation de la RFA s'est enrichie continuellement de clauses restreignant [Ausweisungsschutz et Abschiebungshindernisse] ou suspendant (sursis : Duldung et suspension sur décision d'un Land) l'expulsion. Au fil des amendements, notamment à la loi sur les étrangers et à celle réglementant le droit d'asile, ces articles protégeant dans certains cas les étrangers contre une expulsion hors d'Allemagne ont été précisés. Cela conduit souvent à une extension de cette protection à de nouveaux groupes ou individus étrangers mais aussi à une restriction de ces mêmes possibilités de recours.

Dans le contexte des centres de rétention, les droits de recours font l'objet d'une course poursuite permanente entre d'une part, les détenus et leurs avocats, et d'autre part les autorités des étrangers et les tribunaux compétents. Souvent lorsqu'un recours contre un placement en centre de rétention a abouti devant une juridiction, un usage ultérieur de cette brèche du dispositif est limité par l'utilisation dans les courriers administratifs de formules standards ne pouvant plus faire l'objet de contestation juridique.

Par ailleurs la portée de ces possibilités de pourvoi demeure souvent dans les pratiques assez limitée. Les étrangers placés en centre de rétention ne disposent généralement pas des moyens et capacités matériels requis pour faire valoir un recours ${ }^{21}$. Leurs ressources financières ne sont en général pas suffisantes pour bénéficier de l'aide d'un avocat. L'aide gratuite pour les frais de procédure ne semble pas leur être octroyée facilement. A cela s'ajoute les conditions particulières des étrangers ne parlant ou ne maitrisant pas la langue allemande, qui plus est juridique. En outre, le matériel nécessaire pour mener une correspondance avec les autorités compétentes fait bien souvent défaut (timbres, photocopies d'actes, communications avec le pays d'origine...).

Micro-résistance des expulsables

Les droits de recours sont en définitive des ressources que les étrangers placés en centre de rétention peuvent insérer dans leur stratégie de lutte, leur résistance dans le cadre même de la relation de pouvoir qui s'instaure entre eux, simples individus, et ce que nous avons auparavant identifié comme le dispositif d'expulsion.

Parmi les autres moyens utilisés par les étrangers placés en centre de rétention afin d'obtenir leur libération ou du moins éviter d'être expulsé, le refus de dévoiler son identité, d'aider en quoi que ce soit à son identification (par exemple en refusant d'apposer ses empreintes digitales) constitue l'essentiel de ce que l'on pourrait dénommer une «micro-résistance » au cœur du dispositif d'expulsion.

Ce n'est pas un hasard si justement les autorités des étrangers font de la participation $\mathrm{du}$ détenu aux «mesures d'établissement de son passeport» [Paßbeschaffungsmaßnahmen] la quasi-condition du prolongement du placement en centre de rétention : cette « insoumission » aux injonctions du dispositif apparait pour le détenu comme une des rares possibilités, au milieu de son dénuement, de renverser la situation à son avantage et de bloquer la stratégie du dispositif d'expulsion. Pour effectuer une expulsion, l'Etat de droit exige en effet de disposer d'un minimum d'informations sur la personne qu'il a l'intention d'expulser, sans cela le dispositif s'enraye.

Le dispositif d'expulsion atteint peut-être ici une de ses limites face à une «microrésistance " du détenu, à l'autre extrémité la "macro-résistance " possible d'Etats limite également la capacité du dispositif à remplir son objectif stratégique : «Comme il ne saurait y avoir de relations de pouvoir sans points d'insoumission qui par définition lui échappent, toute intensification, toute extension des rapports de pouvoir pour les 
soumettre ne peuvent que conduire aux limites de l'exercice du pouvoir; celui-ci rencontre alors sa butée soit dans un type d'action qui réduit l'autre à l'impuissance totale (une "victoire » sur l'adversaire se substitue à l'exercice du pouvoir), soit dans un retournement de ceux qu'on gouverne et leur transformation en adversaires ${ }^{22}$.

Par ailleurs il est intéressant de noter que le dispositif d'expulsion repose en grande partie sur l'accumulation de connaissances, un certain "savoir » : le face-à-face entre l'étranger placé en centre de rétention et les différentes agences qui coopèrent en vue de son expulsion est avant tout lié au besoin de ces agences de collecter des informations sur ce détenu (état-civil, âge, nationalité, pays de transit...).

La relation de pouvoir se transforme et évolue au gré des résistances apparaissant jusque-là. Au même titre que les procédures de recours tendent à devenir des impasses à partir du moment où un certain seuil d'utilisation de celles-ci est atteint et surtout si elles permettent au plaignant d'empêcher son maintien en détention et/ou son expulsion, les formes de résistance développées par les individus sont rendues inopérantes par de nouvelles pratiques et, si nécessaire, par de nouvelles normes juridiques. Une étrange course poursuite s'est ainsi engagée entre les autorités fédérales allemandes et les migrants illégaux anonymes. Face à la résistance déployée par ces derniers en dissimulant leur identité et surtout toute marque de leur nationalité (papiers d'identité détruits), le gouvernement allemand a entrepris d'opposer des accords de réadmission avec les pays d'origine permettant le renvoi de personnes dépourvues de passeport ou de carte d'identité dont la nationalité aura été prouvée ou rendue crédible [ "glaubhaft gemacht»] à partir de témoignages, de la langue et des indications de la personne concernée, de certificats d'assurance, de permis de conduire...

\section{EXPULSION ET FÉDÉRALISME}

La pratique de l'expulsion en Allemagne est un dispositif qui présente une dimension supplémentaire par rapport aux dispositifs semblables dans les autres pays européens : l'agencement Etat fédéral/Etat fédéré.

L'expulsion et le placement en centre de rétention sont en effet des pratiques qui, d'une part, présentent des différences non négligeables d'un Land à un autre et d'autre part font l'objet de conflits de compétences entre le Bund et les gouvernements régionaux.

Des pratiques différentes suivant les Länder

Les conditions de placement en centre de rétention sont très variables d'un Land à un autre. La plupart du temps les personnes expulsables sont détenues dans l'enceinte de maisons pénitentiaires " classiques", souvent dans des containers particuliers (comme, par exemple, à Rottenburg au Bade-Württemberg). A Berlin, Brême, dans le Brandebourg et en Rhénanie-Palatinat les centres de rétention sont, en principe, distincts des maisons d'arrêt. Dans ces Länder, le placement en centre de rétention est resté du ressort des Ministères régionaux de l'Intérieur.

De fortes différences de conditions de placement en centre de rétention existent cependant également entre les Länder ayant octroyé à des autorités régionales semblables compétence en la matière. Ainsi le centre de rétention de Prötzel dans le Brandebourg est installé au sein d'un centre pour demandeurs d'asile et il n'est pas ferméz ${ }^{23}$. A quelques kilomètres de là, à Berlin, le centre principal de rétention est, en revanche, clos et la circulation à l'intérieur strictement réglementée. La différence 
majeure entre ce centre de rétention et une prison est que les cellules ne sont, en principe, pas fermées.

Par ailleurs, les autorités des étrangers [Ausländerbehörde] étant des administrations locales ou cantonales, certaines régions ont procédé à une centralisation des procédures d'expulsion alors que d'autres (comme la Rhénanie-Palatinat ou le Schleswig-Holstein) laissent à chaque commune ou canton le soin d'organiser les expulsions.

En Basse-Saxe, le Landeskriminalamt de Hanovre est ainsi chargé de l'exécution des expulsions. En Rhénanie du Nord-Westphalie ce sont les cinq Regierungspräsidium [i.e. à peu près l'équivalent d'une préfecture française] qui en sont chargés ${ }^{24}$. Les réservations de billets d'avion, le transfert, l'information des autres administrations concernées sont ainsi centralisées. Cela permet notamment d'organiser le renvoi collectif de personnes originaires d'un même pays.

Parallèlement a lieu également dans certains Länder un regroupement des personnes expulsables en un seul et même centre de rétention.

<!--SPIP--> L'expulsion comme source de conflit entre le Bund et les Länder

Cette insertion de l'expulsion dans le plus vaste débat fédéral n'est pas nouvelle. Dès sa formation, l'Empire allemand est doté de compétences en matière d'immigration et de police des étrangers, mais sous Guillaume Ier et Guillaume II comme sous la République de Weimar, il n'en fera pas usage, laissant aux Länder toute latitude en la matière ${ }^{25}$. Cela tient, en partie, à l'absence, à l'époque, de police au niveau fédéral. La police était du ressort des Länder et donc le Reich ne disposait pas d'organes propres qui puissent exécuter l'expulsion du Reich (Reichsverweisung) ${ }^{26}$. Les autorités fédérales devaient dans ce cas utiliser les autorités policières des Länder.

L'expulsion d'étrangers signifie aussi pour les Länder que leur champ d'action s'élargit ainsi à la scène internationale. Par le biais des procédures d'expulsion ils deviennent des acteurs internationaux à part entière, traitant et négociant avec les pays d'origine ou de transit des expulsés.

Cette projection des entités infra-nationales allemandes au rang d'acteurs internationaux n'est d'ailleurs pas nouvelle. Dès la fin du XIXe siècle, les autorités policières du Land obtinrent en effet compétence pour entrer, par voie diplomatique, en contact direct avec les autorités étrangères à propos de l'admission [Übernahme] d'une personne à expulser ${ }^{27}$. Aujourd'hui les Länder disposent de la possibilité de suspendre l'exécution d'arrêtés d'expulsion d'étrangers d'un pays précis ou d'un groupe d'étrangers en général ou bien leur expulsion vers des pays particuliers (article 54 de la loi allemande sur les étrangers). Cette suspension ne peut pas excéder 6 mois sans l'accord du Ministre fédéral de l'Intérieur. C'est cette limitation imposée à la marge de manœuvre des Länder en matière d'expulsion qui a suscité ces dernières années plusieurs différends entre l'Etat fédéral et les Länder.

Au printemps 1995 le Ministre fédéral de l'Intérieur, Manfred Kanther refusa ainsi publiquement de donner son aval à un prolongement supplémentaire de la suspension des expulsions vers la Turquie de Kurdes et de chrétiens syriens-orthodoxes. Cette suspension avait été décidée un an auparavant par plusieurs Länder, dirigés par les sociaux-démocrates, et elle avait été plusieurs fois prolongée (de mois en mois : mijanvier, mi-février jusqu'à mi-mars 1995) par le Ministre fédéral. L'argumentation de ce dernier était que les Länder, en s'en tenant à une suspension des expulsions pour les 
Kurdes, remettaient en cause le compromis de 1993 sur le droit d'asile ${ }^{28}$, la suspension de l'expulsion de demandeurs d'asile déboutés faisant de l'Allemagne un pays d'immigration (Zuwanderungsland).

Il n'est pas clairement établi que l'Etat fédéral dispose du droit d'obliger les Länder à renoncer à une suspension des expulsions ${ }^{29}$. Dans ce cas précis des demandeurs d'asile kurdes déboutés, le Bund ne déposa aucun recours devant le Tribunal constitutionnel fédéral, mais des jugements au cas par cas reconnurent la nécessité de l'accord du Ministère fédéral de l'Intérieur et invalidèrent la suspension d'expulsions décrétée ${ }^{30}$.

Les divergences et le conflit ne mirent pas seulement aux prises Bund et Länder mais aussi des Länder entre eux suivant leur affiliation politique (la Bavière et la Hesse par exemple). Par ailleurs, les Länder avaient décrété des suspensions d'expulsions fort différentes : certains prônaient de limiter cette suspension aux Kurdes originaires des régions de Turquie où l'état d'urgence est proclamé (c'est-à-dire 10 provinces de l'est et du Sud-Est de la Turquie), d'autres - ou parfois les mêmes - d'en exclure les Kurdes condamnés pour des délits [strafbarer Handlungen].

\section{EXPULSION : UN DISPOSITIF TRANSNATIONAL}

Une des particularités du dispositif de l'expulsion est qu'il est ancré dans un cadre non strictement stato-national. Les différents accords d'expulsion - de "réadmission » conclus, par exemple, par l'Allemagne avec des pays d'origine ou de transit de migrants illégaux donnent une première mesure de cette dimension internationale du dispositif.

L'entrelacement des politiques intérieure et extérieure ne saurait cependant se réduire à ces accords, le plus souvent bilatéraux et somme toute assez classiques au regard des relations internationales. La singularité du dispositif d'expulsion par rapport à d'autres dispositifs est qu'il lie des entités, des acteurs traditionnellement confinés au cadre national ou, au contraire, conçus comme des agents internationaux. En cela le dispositif d'expulsion, tel qu'illustré ici par l'Allemagne, est le lieu par excellence d'une politique transnationale qui s'étend toujours plus avant vers l'extérieur et l'intérieur des Etatsnations.

Lire la suite

Revenir à la partie précédente

\section{NOTES}

1. Deutscher Bundestag, Drucksache IV/868, "Entwurf eines Gesetzes über den Aufenthalt der Ausländer (Ausländergesetz)", pp.15-16.

2. Deutscher Bundestag, Drucksache IV/868, "Entwurf eines Gesetzes über den Aufenthalt der Ausländer (Ausländergesetz)", p.16.

3. «Contre le renvoi, la reconduite à la frontière et contre l'ordre de placement en centre de rétention [Abschiebungshaft] aucune voie de recours n'est prévue. » Art. 11, Abs. 5, Ausländerpolizeiverordnung (22.08.1938), Reichsgesetzblatt, Teil I, 25.08.1938, Nr.132, p.1055. 
4. « (3) La détention de sûreté [Sicherungshaft] peut être ordonnée pour une durée de six mois. Elle peut, dans le cas où l'étranger empêche son expulsion, être prolongée au maximum de douze mois (...) » §57. Abschiebungshaft. Ausländergesetz. 9. Juli 1990. BGBl. I p.1354.

5. Cf. Jörg Wegner, "Die Abschiebungshaft auf dem verfassungsrechtlichen Prüfstand", Zeitschrift für Ausländerrecht und Ausländerpolitik [ZAR], Heft 2, 25. April 1996, p. 78.

6. Voir Abgeordnetenhaus von Berlin, 12. Wahlperiode, Drucksache 12/5879.

7. Senatsverwaltung für Inneres (Berlin), Bericht über die Maßnahmen zur Verbesserung der Situation im Abschiebungsgewahrsam, Nov. 1994, p.6.

8. Abgeordnetenhaus von Berlin, 12. Wahlperiode, Drucksache 12/5879 et 12/5989. 9. « ... l'essentiel de la peine (...) ne croyez pas qu'il consiste à punir ; il cherche à corriger, redresser, "guérir". (...) une peine qui, si elle sanctionne le crime, peut se modifier (s'abrégeant ou, le cas échéant, se prolongeant) selon que se transforme le comportement du condamné ; elles le sont encore par le jeu de ces "mesures de sûreté" dont on accompagne la peine (interdiction de séjour, liberté surveillée, tutelle pénale, traitement médical obligatoire) et qui ne sont pas destinées à sanctionner l'infraction, mais à contrôler l'individu, à neutraliser son état dangereux, à modifier ses dispositions criminelles, et à ne cesser qu'une fois ce changement obtenu. » Michel Foucault, Surveiller et punir, naissance de la prison, Paris, Gallimard, 1975, pp.17 et 25-26. 10. Michel Foucault, Surveiller et punir, naissance de la prison, Paris, Gallimard, 1975, p.25.

11. Initiative gegen Abschiebehaft (Hrsg.), Abschiebungshaft Berlin - Endstation Deutschland ? !, Berlin, Juli 1995, p.21.

12. Cf. concernant Büren : Flüchtlingsrat NRW, Sonderthemenheft : Abschiebehaft, Dülmen, 1994, p.7.

13. Senatsverwaltung für Inneres (Berlin), Bericht über die Maßnahmen zur Verbesserung der Situation im Abschiebungsgewahrsam, Nov. 1994, p.8.

14. Cf. Eckart Spoo, "Abschiebehäftlinge sollen zahlen", Frankfurter Rundschau, 29.11.1994 \& "Abschiebehaft. Niedersachsen verzichtet jetzt auf Kostenerstattung", Frankfurter Rundschau, 7.12.1994 ; Frank Siering/Susann Hellwig, "116 Mark pro Nacht", Focus, 1994, Nr. 49.

15. Cf. "Gespräche über Rückführung abgelehnter Asylbewerber geplant", Frankfurter Allgemeine Zeitung, 31 janvier 1996, p.5.

16. James F. Hollifield, "Entre droit et marché", in Bertrand Badie/Catherine Wihtol de Wenden (dir.), Le défi migratoire, questions de relations internationales, Paris, Presses de la Fondation Nationale des Sciences Politiques, 1994, p.60.

17. Hollifield, op. cit., p.63.

18. Hollifield, op. cit., p.62.

19. Cf. supra et $\$ 54$ PVG [Polizeiverwaltungsgesetz] i.V.m. VO über Rechtsmittel in Angelegenheiten der Ausländerpolizei vom 1.10.1931 cité in Götz, op. cit., p.412.

20. Cf. par exemple l'absence de tout recours contre le placement en Schutzhaft, dans l'art. 7 de la loi sur la Gestapo de 1936 : «Les dispositions du domaine de la police secrète d'Etat [Geheime Staatspolizei] ne sont pas sujettes à un examen par les tribunaux administratifs. »

21. Cf. par exemple dans le contexte particulier du "règlement d'aéroport" [Flughafenregelung] des demandes de droit d'asile, les réserves exprimées par le Tribunal constitutionnel fédéral à propos de la protection judiciaire effective dans son arrêt du 14 mai 1996 (2 BvR 1516/93). 
22. Michel Foucault, "Le pouvoir comment s'exerce-t-il ?", op.cit., pp.319-320.

23. Un quart des personnes expulsables retenues prendraient en conséquence la fuite.

24. Il s'agit des autorités centrales des étrangers [ZAB : Zentralen Ausländerbehörden], Cf. Peter Knösel, Freiheitlicher Rechtsstaat und Abschiebung, Berlin, Berlin Verlag/

Arno Spitz, 1991, p.52, 55 et 88.

25. Cf. Werner Kobarg, Ausweisung und Abweisung von Ausländern, Berlin, Dr. Walther Rothschild, 1930, p.49; Volkmar GÖTZ, "Polizei und Polizeirecht", in Kurt G.A.

JESERICH, Hans POHL, Georg-Christoph von UNRUH (Hrsg.), Deutsche

Verwaltungsgeschichte, Band 4 : Das Reich als Republik und in der Zeit des Nationalsozialismus, Stuttgart, Deutsche Verlags-Anstalt, 1985, p.411.

26. L'organe éxécutif de l'Empire était l'armée. Cf. Kobarg, op. cit.., p.50 et Götz, op. cit., p.398.

27. Vorschriften des Bundesrates betreffs die Vollziehung der Ausweisung (10 décembre 1890).

28. Cf. "Über die Abschiebung von Kurden heftige Auseinandersetzungen im Bundestag", Frankfurter Allgemeine Zeitung, 18 mars 1995, p.1.

29. Cf. Ralph Göbel-Zimmermann, "Handlungsspielräume der Landesregierungen für den Erlaß von Abschiebungsstoppregelungen", Zeitschrift für Ausländerrecht und Ausländerpolitik, 10 janvier 1995, p.28.

30. Voir, par exemple, un arrêt du Tribunal administratif de Hesse du 27 juillet 1995 déclarant contraire à la loi la suspension d'expulsions décidée le mois précédent par le Ministère de l'Intérieur de Hesse en faveur des Kurdes de Turquie, Cf.

"Abschiebungsstopp des Landes Hessen gesetzwidrig", ZAR Aktuell, Nr. 4/1995, 28 juillet 1995, p.1.

INDEX

Index géographique : Allemagne

Mots-clés : contrôle, étrangers, expulsions 